\title{
Can the Double-J Ureteric Stent be Dispensed? A Prospective Randomized Study in Renal Transplant Recipients
}

Murthy PVLN*, Ramreddy CH, Ramachandraiah G, Kiran Kumar D, Vidyasagar S and Devraj R Department of Urology and Renal Transplantation, Nizam's Institute of Medical Sciences, India

*Corresponding author: Murthy PVLN, Department of Urology and Renal Transplantation, Nizam's Institute of Medical Sciences, 202,10-2-289/12, Sripadaresidency,

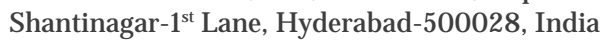

Received: March 12, 2017; Accepted: April 18, 2017; Published: May 03, 2017

\begin{abstract}
The use of prophylactic double-J ureteric (DJ) stent during renal transplantation is debatable. The authors who favor stenting claim the incidence of urological complications and morbidity were less. On the other hand, literature shows routine stenting is unnecessary as it adds to the cost and complications. With this background, we performed a prospective randomized controlled study in live related renal transplant recipients with and without a DJ stent to know whether it can be avoided.
\end{abstract}

Seventy-six consenting patients for live related renal transplantation were recruited in this study between November 2014 to August 2015 at our centre. Patients were randomized in to two groups, group A with DJ-stent and group $B$ without a stent based on computer randomization. These patients were evaluated in the immediate and at the end of $4,12 \& 24$ weeks post operatively for urological complication. Urine culture, serum creatinine, ultrasound and Doppler examination of the graft were performed as per the protocol. DJ- stent was removed at the end of 4 weeks.

Thirty six patients in group A with stent and 36 in group B without a stent were evaluated for urinary tract infection, urinary leak and ureteric obstruction postoperatively and found no statistical difference between the two groups. Four patients were excluded

Routine use of DJ- stents may not be indicated during Kidney transplantation. Careful surgical technique with selective stenting of problematic anastomoses yields similar results. The incidence of UTI is comparable in both groups.

Keywords: Renal transplantation; Urological complications; DJ-Stent; Urine leak

\section{Abbreviations}

DJ Stent: Double J Stent; UTI: Urinary Tract Infection

\section{Introduction}

In renal transplantation, the use of DJ - stents to prevent postoperative complications like urine leaks, obstruction or strictures is well known [1]. But however there is controversy in placement of DJ- stents during renal transplantation, as observed in retrospective [2] and prospective randomized trials as well [3].

Proposed benefits to a stented anastomosis include continuous decompression of the ureter to avoid anastomotic tension, maintenance of the ureter in a more linear alignment to avoid kinking and protection from ureteral narrowing or postoperative ureteric obstruction due to edema or external compression [4].

Routine use of DJ stents in an immunosuppressed transplant recipient, places him or her at high-risk for development of complications like urinary tract infection (UTI), stent encrustation and stone formation. The incidence of UTI is not only higher in the immediate postoperative period, but also after removal of the stents
[5]. Placing a DJ stent means, it has to be enrolled in a stent registry to avoid the possibility of a retained or forgotten stent. Extra cost involved in removal of the stent by cystoscopy and additional need for anesthesia should be considered in these immunocompromised individuals, especially in children [6].

The aims and objectives of our study was to evaluate the incidence of UTI, Urinary leak and obstruction with and without a DJ stent during ureteroneocystostomy and whether a DJ stent can be avoided.

\section{Materials and Methods}

After obtaining ethics committee approval, the study was carried out from November 2014 to August 2015 at our centre. Seventy six consecutive patients were enrolled into this study after taking informed consent. They were randomized into two groups of 38 each. In Group A DJ stent was placed and Group B was without a stent based on computer generated random numbers using Rand between function of Microsoft Excel.

All patients underwent live related donor renal transplantation in which the kidney was procured by open donor nephrectomy and extravesical ureteroneocystostomy by Lich-Gregoir technique with or 
Table 1: Patient characteristics.

Table 1: Patient characteristics.
\begin{tabular}{|c|c|c|c|}
\hline Variable & Group A & Group B & P value \\
\hline Age (years) & $31.78 \pm 10.17$ & $29.09 \pm 8.96$ & 0.239 \\
\hline Gender M/F & $31: 5$ & $30: 6$ & \\
\hline Graft survival (6 months) & $100 \%$ & $100 \%$ & \\
\hline
\end{tabular}

without DJ (double J) stent. A 6-0 double arm (13 mm) PDS suture was used for the anastomosis.

Urethral Foleys catheter was removed on $3^{\text {rd }}$ and wound drain on $4^{\text {th }}$ post operative days. DJ stent was removed cystoscopically 4 weeks after surgery.

Urine samples for culture were collected preoperatively, postoperatively on day 3, (after removal of Foley's catheter), 4 weeks (after surgery before removal of DJ stent), 3months post op and whenever needed depending upon the patients' symptomatology (fever, dysuria). Serum creatinine: (normal: 0.7-1.5 mg) evaluated before discharge, at 1 month, 3 months and 6months respectively.

Ultrasonography was performed on $3^{\text {rd }}$ postoperative day to detect any perinephric collection and ureteric obstruction along with Doppler for graft vascularity (as a protocol) and Graft ultrasonography at three months follow up.

Statistical Analysis was done using SPSS version 17. Continuous variables were represented as mean with standard deviation (SD) and categorical variables as numbers with percentages. Continuous variables are compared between both the groups using independent sample t-test and categorical variables are compared using Chi-square test and Fisher exact test when expected cell value is less than 5 .

\section{Results}

Between November 2014 to August 2015, 76 consecutive live related renal transplants were enrolled into the study of which four patients were excluded. The reasons being one patient (with DJ stent, group-A) died of pseudomonas sepsis on $7^{\text {th }}$ post op day. The source of infection was proved to be internal jugular vein catheter and the graft function was normal at the time of death.

The second patient developed ureteric leak (group-B) due to ureteral ischemia which required exploration and repeat ureteroneocystostomy with a DJ stent after excising the ischemic ureter. In the $3^{\text {rd }}$ patient, diuresis was delayed (group-B) and required stenting. Fourth patient (group A) underwent graft nephrectomy for renal artery thrombosis on $7^{\text {th }}$ post op day.

Finally 72 patients 36 in each group were analyzed. Group A $(n=36)$ with a DJ stent and Group-B $(n=36)$ without a stent (Table 1) summarized the characteristics of the patient population.

Mean value of serum creatinine in stented group is 1.308 and in unstented group is 1.37 ( $\mathrm{p}=0.609$ ) which is not statistically significant (Table 2).

Table 2: Serum creatinine levels in both groups.

\begin{tabular}{|c|c|c|c|c|c|c|c|c|c|}
\hline \multirow{2}{*}{ Parameter } & \multicolumn{4}{|c|}{ Group A } & \multicolumn{4}{|c|}{ Group B } & \multirow[t]{2}{*}{$P$ value } \\
\hline & Before discharge & I month & 3 month & 6 month & Before discharge & 1 month & 3 month & 6 month & \\
\hline S. $C r<1.5$ & 33 & 32 & 32 & 33 & 31 & 30 & 31 & 32 & \multirow{2}{*}{0.609} \\
\hline S. $\mathrm{Cr}>1.5$ & 3 & 4 & 4 & 3 & 5 & 6 & 5 & 4 & \\
\hline
\end{tabular}

\begin{tabular}{|c|c|c|c|c|}
\hline & POD-5 & $1^{\text {st }}$ MONTH & $3^{\text {rd }}$ MONTH & P value \\
\hline GROUP A & 2 pts. & 2 pts. & 5 pts. & \\
\hline GROUP B & 2 pts & 3 pts & 3 pts & 0.129 \\
\hline
\end{tabular}

\section{Urinary tract infection (UTI)}

Overall, there was no difference in the incidence of UTI between the two groups. Even though there was a higher incidence of UTI in stented group (25\%) compared to unstented group (20\%) p value was 0.129 which was not statistically significant (Table 3 ).

Causative organisms were similar in both groups, of which E coli was most common. None of our patient developed pyelonephritis.

\section{Obstruction/leak}

No case of anatomotic urinary leak occurred in either group. Although there were no urologic complications in the stent group $(\mathrm{N}=36), 2$ patients $(5 \%)$ had ureteric dilatation in unstented group. However there was no evidence of obstruction as serum creatinine and renogram study were normal. None of the patients in Group B underwent any intervention for urological complications.

\section{Discussion}

Use of routine ureteric stents during uretronoecystostomy in renal transplant recipients is debatable. Our study results clearly showed that there was no statistically significant difference in urological complications between the two groups. The incidence of UTI was $25 \%$ (9 pts.) in the stented group and $20 \%$ (8 pts) in unstented group in 3 months follow up which is not statistically significant (Table 3). The rate of UTI in transplanted patients with stented ureteroneocystostomy has been reported to be as high as $31 \%$ [7]. Others reported no significant difference in the rate of UTI between stented and unstented patients $[4,8]$. In our study although there was a trend towards a higher incidence of UTI within the first 3 months post transplant we found no difference in the incidence of UTI after 6 months.

In our study, the overall rate of urologic complications like obstruction and anastomotic urinary leak in unstented group were nil. Although two patients (5\%) showed ureteric dilatation in group B there was no obstruction as renogram and the serum creatinine levels were normal. Non obstructed ureteric dilatation is not uncommon in post transplant setting due to increased diuresis and this can be confirmed by serial serum creatinine estimation and DTPA renogram. One patient in group A who developed urine leak on $7^{\text {th }}$ post op day was excluded from the study because of ureteric leak due to ischemia. This patient developed a large pelvic hematoma and hemodynamically unstable on $2^{\text {nd }}$ post op day. Exploration revealed a bleeding vessel from the perinephric fat of the graft and the hematoma was compressing the ureter. The hematoma was drained and homeostasis was secured. But he developed a urine leak on $7^{\text {th }}$ post op day and exploration showed the terminal $3 \mathrm{~cm}$ of
Table 3: Urinary Tract Infection (culture Positive). 
ureter ischemia with a urine leak from a perforated terminal ureter. This could be due to compression by the hematoma and /or post op hypotension resulting in ischemic perforation of the terminal ureter. This patient was in unstented group and even a prior DJ-stent could not have prevented urinary leak from the ischemic ureter.

In a prospective study by Kumar, et al. the authors concluded that routine placement of ureteric stent was cost effective and almost eliminated urological complications [4]. It has been reported that routine use of DJ stents in renal transplantation significantly reduced the number of urinary leaks and ureteral obstruction $[8,9]$. Srivasthava, et al. reported $7.7 \%$ complication rate with nonstented and 2\% with stented ureteral anastomosis [10]. Studies reported urologic complication rates as high as 13\% in unstented renal transplant recipients [11]. Others suggest routine use of stents to lower this rate to less than $8 \%$ [8]. Other trials could not identify an advantage to routine stenting [12] and concluded that selective stenting yields complication rates similar to those seen when stents are used routinely.

The ureteric leaks and obstruction ultimately depends on how well the ureter is harvested preserving its vascularity, delicate handling of tissues and technique of ureteroneocystostomy. Small to moderate anastomotic urinary leaks will subside with no consequence in the presence of a stent, but ischemic ureteric leak will not respond in spite of a stent in situ. Careful harvesting of ureter during donor nephrectomy is of paramount importance. There is no substitute for a careful and meticulous surgical technique. We feel that ureteroneocystostomy should be performed with same precision and caution as we do vascular anastomosis. The 'Golden triangle' should not be disturbed while harvesting the ureter and the laminapropria which contains the vascular supply to the underlying mucosa is not to be damaged while making sub mucosal tunnel in the bladder.

Although the risk of urological complications following renal transplantation has decreased over the last several years, the controversy regarding the use of ureteral stents continues.

The ideal timing of stent removal post-transplantation is a contentious issue. There is no declared optimal time for the removal of DJ stent. The removal time was reported between postoperative first week and 3 months in some of the reports of transplant centers, according to their protocols. It was reported that the incidence of stent related complications including UTI, hematuria, stent encrustation and lower urinary tract symptoms were less if the stents were removed on day 30 after transplantation [13,14]. So stent removal within 4 weeks of insertion appears advisable. Prophylactic antibiotics in post transplant patients reduce the incidence of stent related UTI or pyelonephritis. We observed the incidence of stent related UTI was very much reduced while transplant patients were put on trimethoprim sulfamethoxazole prophylaxis for pnuemocystis Carinii.

\section{Conclusion}

Routine use of DJ stents may not be indicated during Kidney transplantation. Careful surgical technique with selective stenting of problematic anastomoses yields similar results. There is no increased incidence in UTI in stented group.

\section{References}

1. Shokeir A, el-Diasty T, Ghoneim M. Endourologic management of ureteric complications after live-donor kidney transplantation. J Endourol. 1993; 7 : 487-491.

2. Acott P, Crocker J, Bitter-Suermann H, Lawen JG. Extravesical ureteroneocystostomy with and without internalized ureteric stents in pediatric renal transplantation. Pediatr Transplant. 2001; 5: 21-26.

3. Pleass H, Clark K, Rigg KM, Reddy KS, Forsythe JL, Proud J, et al. Urologic complications after renal transplantation: a prospective randomized trial comparing different techniques of ureteric anastomosis and the use of prophylactic ureteric stents. Transplant Proc. 1995; 27:1091-1092.

4. Kumar A, Kumar R, Bhandari M. Significance of routine JJ stenting in living related renal transplantation: A prospective, randomized study. Transplant Proc. 1998; 30: 2995-2997

5. Ranganathan M, Akbar M, Ilham MA, Chavez R, Kumar N, Asderakis A. Infective complications associated with ureteral stents in renal transplant recipients. Transplant proc. 2009; 41: 162-164.

6. Darnidharka VR, Araya CE, Wadsworth CS, McKinney MC, Howard RJ. Assessing the value of ureteral stent placement in pediatric kidney transplant recipients. Transplantation. 2008; 85: 986-991.

7. Koo Seen Lin LC, Bewick M, Koffman CG. Primary use of ureteral stents in renal transplantation. Br J Urol 1993; 72: 697-701.

8. Rodriguez GV, Martinez RM, Arguinarena TF, Martine MS, De Castro Olmedo C, Del Busto FE. The use of double J stent or prevention of urological complications in kidney transplants. Acta Urol Esp 2008; 32: 225-229.

9. Shum CF, Lau KO, Cheng WS. Urological complications in renal transplantation. Singapore Medical J 2006; 47: 388-391.

10. Srivastava A, Choudhary H, Sehagal A, Dubey D, Kapoor R, Kumar A. Ureteric complications in live related donor renal transplant: Impact on graft and patient survival. Indian J Urol. 2004; 20: 11-14.

11. Benoit G, Blanchet P, Eschwege L, Alexandre L, Bensadoun H, Charpentier B. Insertion of a double pigtail ureteral stent for the prevention of urological complications in renal transplantation: a prospective randomized study. J Urol. 1996; 156: 881-884.

12. Bassiri A, Amiransari B, Yazdani M, Sesavar $Y$, Gol S. Renal transplantation using stents. Transplantation Proc. 1995; 27: 2593-2594.

13. Tavakoli A, Surange RS, Pearson RC, Parrott NR, Augustine T, Riad HN. Impact of stents on urological complications and health care expenditure in renal transplant recipients: results of a prospective, randomized clinical trial. J Urol. 2007; 177: 2260-2264.

14. DuBay DA, Lynch R, Cohn J, Ads Y, Punch JD, Pelletier SJ, et al. Is routine ureteral stenting cost- effective in renal transplantation? J Urol. 2007; 178 2509-2513.
Austin J Nephrol Hypertens - Volume 4 Issue 1 - 2017 ISSN : 2381-8964 | www.austinpublishing group.com Murthy et al. (C) All rights are reserved
Citation: Murthy PVLN, Ramreddy CH, Ramachandraiah G, Kiran Kumar D, Vidyasagar S and Devraj R. Can the Double-J Ureteric Stent be Dispensed? A Prospective Randomized Study in Renal Transplant Recipients. Austin J Nephrol Hypertens. 2017; 4(1): 1063. 